\title{
Water-driven stabilization of diphenylalanine nanotube structures
}

\author{
T. Andrade-Filho ${ }^{1}$ 'Tiago Carvalho Martins ${ }^{1} \cdot$ Fabio Furlan Ferreira $^{2} \cdot$ \\ Wendel Andrade Alves ${ }^{2} \cdot$ Alexandre Reily Rocha $^{3}$
}

Received: 10 May 2016 / Accepted: 20 June 2016 / Published online: 11 July 2016

(C) Springer-Verlag Berlin Heidelberg 2016

\begin{abstract}
L, L$-diphenylalanine has been employed in the formation of self-assembled peptide nanotubes with great potential for the development of biosensors, molecular carriers, and optoelectronic devices. They are usually formed in an aqueous solution, and it is well known that water remains confined inside the structure. However, the role played by water in the overall stability of the nanotube is still unknown at the microscopic level. In this work, we investigate the stability of peptide structures after assembly due to the interaction with water molecules. We demonstrate, using molecular dynamics based on density functional tight-binding techniques, that water is fundamental in keeping the nanotube structure. It interacts with the nanotube walls as well as with other water molecules via hydrogen bonds keeping the structure stable. We identify and quantify the interaction between water and the relevant groups, and, upon increasing the solvent concentration, we show there is a transition region where there is a competition between the formation of water/water hydrogen bonds, and steric effects.
\end{abstract}

Keywords Peptide nanotubes - Structural stability $\cdot$ Water properties $\cdot$ DFTB

T. Andrade-Filho

tarcisofilho@unifesspa.edu.br

Alexandre Reily Rocha

reilya@ift.unesp.br

1 Faculdade de Física, Universidade Federal do Sul e Sudeste do Pará (UNIFESSPA), 68505-080 Marabá, Pará, Brazil

2 Centro de Ciências Naturais e Humanas, Universidade Federal do ABC, 09210-180 Santo André, São Paulo, Brazil

3 Instituto de Física Teórica, Universidade Estadual Paulista (UNESP), 01140-070 São Paulo, Brazil

\section{Introduction}

Self-assembled peptides have attracted great attention in the last years, because they serve as building blocks that can provide biocompatibility, chemical versatility, biological recognition abilities, and facile synthesis $[1,13]$. Since Ghadiri et al. [17] described a new class of organic nanotubes based on a cyclic peptide architecture, these structures have been used for the assembly of a variety of nano-ordered materials. A number of peptide-based nanomaterials have been prepared using different building blocks, including cyclic peptides with alternating D- and L- amino acids [17, 23], peptide amphiphiles and peptide bolaamphiphiles [10], short peptides with alternating negatively and positively charged residues, and surfactant-like peptides [42], aromatic dipeptides [35], and hydrophobic dipeptides [18]. The molecular sequence can be carefully selected based on the expected interactions between the specific amino acids, such as $\beta$-sheet, aromatic stacking or alternating hydrophilic and hydrophobic interactions [51]. In fact, the range of choices and the versatility is what provides the potential for a wide range of applications, from biosensors $[6,30,48]$ to energy storage devices $[38,50]$.

One of the shortest biologically active peptides that can self-assemble is $L, L$-diphenylalanine (FF), the Alzheimer's $\beta$-amyloid polypeptide motif. There are reports of several types of ordered nanostructures such as nanoribbons [22], nanovesicles [46], nanotubes [28, 35], and nanowires based on FF [21, 28]. Ordering occurs at different scales starting at the molecular level with the association of six FF monomers forming a macrocycle, as illustrated in Fig. 1a. There, amino and carboxyl groups form the inner core of the cycle creating a hydrophilic cavity that hosts $\mathrm{H}_{2} \mathrm{O}$ molecules [28]. The macrocycles stack into a columnar phase forming 


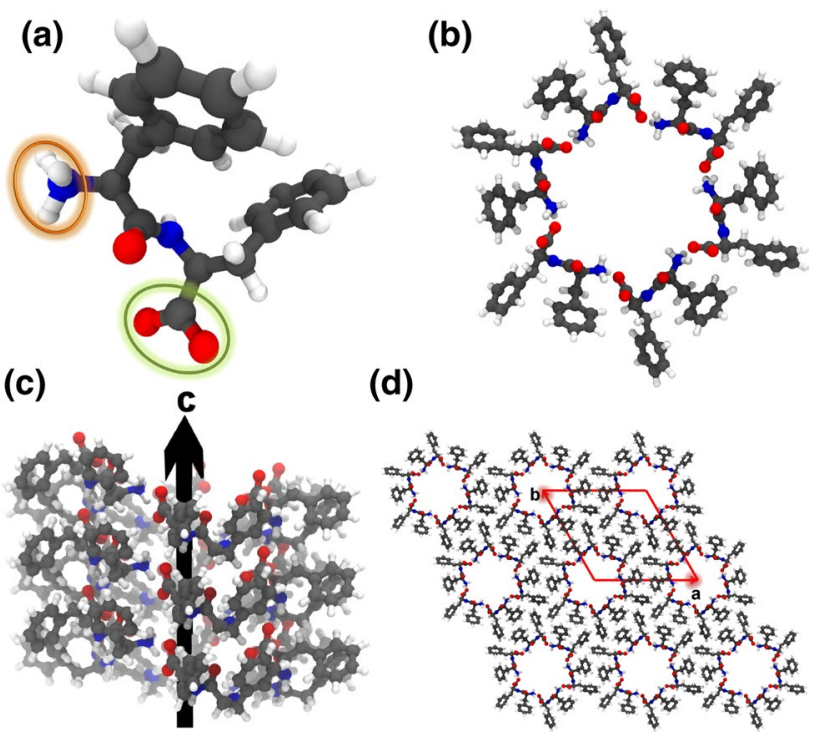

Fig. 1 a A single FF zwitterionic molecule, b hexamer building block unit of six FF molecules, c vertical stacking of hexamer units forming a single NT, d lateral stacking of isolated NTs showing the hexagonal packing of the crystal system. The bright orange and green ellipses refer to ammonium $\left(\mathrm{NH}_{3}^{+}\right)$and carboxylate $\left(\mathrm{COO}^{-}\right)$groups

narrow tubes with diameter $\sim 1 \mathrm{~nm}$. These nanotubes can further pack to form a hexagonal arrangement and give rise to pleated sheets [36]. Subsequently, through successive associations, create fibrillar bundles. The crystallographic arrangement has been appropriately described according to the hexagonal symmetry group $\mathrm{P} 6_{1}$ for self-assembly occurring in an aqueous environment $[8,28]$.

A number of works have focused on the self-assembly process of supramolecular FF-based structures-including nanotubes-using both theory [20, 26, 27] and experiments $[11,28,43,45]$. In all of them, the role played by the aqueous solution is an important aspect. In fact, Kim et al. [28] have shown, for example, that different concentrations of water in the precursor solution lead to the formation of either nanotubes or nanowires. Furthermore, molecular dynamics simulations indicate that the zwitterionic form (one $\mathrm{NH}_{3}^{+}$and one $\mathrm{COO}^{-}$per FF shown in Fig. 1) of the peptide is more prone to self-assembling nanotubes when compared to its neutral form [26]. Once the FFNT has been synthesized, channels with hydrophilic character are observed [19]. Thus, during the wrapping process, water molecules present in the solution are confined inside the channel $[28,35]$. These molecules are known to give rise to changes in the electronic structure of the system $[4,45]$.

Nevertheless, the part water plays in the stability of the tubular arrangement after it has been formed is not yet clear. Typically, in a hydrated medium, zwitterionic molecules are more stable than the neutral forms [25, 29, 41]. It is known that the removal of water in $L, L$-diphenylalanine isolated peptides leads to a linear to cyclic transition [2]. Görbitz [19] proposed that, in order to stabilize the zwitterionic structure, the extra hydrogen atom of the $\mathrm{NH}_{3}^{+}$ groups would be hydrogen bonded to water molecules inside the nanotube, i.e., confined water would not just be a consequence of the synthesis, but a requirement for stability. It is not clear however, whether once formed the tubes would be stable even if water were removed or whether the peptides would undergo a zwitterionic/neutral form transition. In fact, an irreversible phase transition from the hexagonal to an orthorhombic P22 $2_{1}{ }_{1}$ arrangement is observed at $150{ }^{\circ} \mathrm{C}$, an indication that water is key not only to the formation of the tubes, but also to their stability afterwards $[2,29,40]$.

In order to study the role of water inside the nanotube, and the mechanisms driving their stability, we employed the dispersion-corrected third-order self-consistent charge density functional tight-binding (DFTB3) method to model the crystal structure of FFNTs with and without solvent molecules trapped in the hydrophilic channel [15]. The temperature effects were taken into account using DFTB3 molecular dynamics (DFTB3-MD) simulations. We show that the removal of water from inside the tubes leads to a zwitterionic to neutral transition that renders the whole structure unstable. The inclusion of water molecules stabilizes the system, and that stability is driven by a combination of hydrogen bond formation between water molecules and the walls of the NT, as well as HBs within water molecules.

\section{Theoretical calculations}

All the calculations were performed with a four-step process using the DFTB3 method as it yields a good balance between accuracy and efficiency [15]. It is a third-order Taylor expansion of the Kohn-Sham energy functional with respect to charge density fluctuations. Compared to its predecessor [12], the description of hydrogen bonding is enhanced by changing the behavior of the screened Coulomb interaction at short distances, when a hydrogen atom is involved in the interaction $[15,16]$. All the calculations were performed with the DFTB+ package [5].

First, the crystal structure of the anhydrous FF nanotube shown in Fig. 1 was relaxed using the conjugate gradient $(\mathrm{CG})$ method with an atomic force tolerance of $1.0 \times 10^{-3} \mathrm{eV} / \AA^{-1}$. The anhydrous and non-centrosymmetric hexagonal unit cell ( $\mathrm{P}_{1}$ space group) contains six FF molecules, and it is formed by a total of 258 atoms [8, 28]. The starting configurations were obtained from X-ray experimental data [8]. In all cases, the Brillouin zone was sampled using a Monkhorst-Pack [34] scheme with a $2 \times 2 \times 3$ k-point sampling. 
In a second step, water molecules, in different concentrations, were confined in the central hydrophilic channel of the nanotube (varying from one to 23 molecules per hexamer). They were inserted in the channel using a boxconstrained algorithm implemented in the Packmol program [32, 33]. Later, the crystal structures of the complex (NT plus water) were relaxed (with parameters similar to the CG method).

Subsequently, a DFTB3-MD simulation was performed taking into account a simulated annealing protocol. The calculation was carried out in the NVT Ensemble using the Andersen thermostat [3]. The structures were heated from 0.0 to $353.0 \mathrm{~K}$ during $2.0 \mathrm{ps}$, and then kept at $353.0 \mathrm{~K}$ for an extra $2.0 \mathrm{ps}$. The system was then cooled to room temperature in $2.0 \mathrm{ps}$. The production run at room temperature had a total duration of $10.0 \mathrm{ps}$. After that, each structure was cooled to $0.0 \mathrm{~K}$ within a time window of up to $6.0 \mathrm{ps}$. The procedure was repeated for four different configurations for sampling. The integration of equations of motion was performed using the Verlet velocity algorithm with a time step of $0.5 \mathrm{fs}$.

The fourth step corresponds to the CG relaxation of each structure. Using the final atomic arrangements, we calculated the average binding energy per FF hexamer as a function of the number of water molecules,

$E_{b}(n)=\frac{1}{4} \sum_{i=1}^{4}\left(E_{\text {complex }}^{n}(i)-E_{\mathrm{NT}}-n E_{\text {water }}\right)$,

where $E_{\text {complex }}^{n}(i), E_{\mathrm{NT}}, E_{\mathrm{water}}$ and $n$ correspond to the energy of the $n$th complex (nanotube $+n$-water molecules), energy of the anhydrous nanotube, energy of a relaxed single water molecule, and the number of $\mathrm{H}_{2} \mathrm{O}$ molecules used in the calculation at $0.0 \mathrm{~K}$, respectively. We also calculated the coupling energy,

$E_{c}(n)=\frac{1}{4} \sum_{i=1}^{4}\left(E_{\text {complex }}^{n}(i)-E_{\mathrm{NT}}-E_{\text {water-isol }}^{n}(i)\right)$,

where $E_{\text {water-isol }}^{n}(i)$ is the energy of an isolated tube of water molecules containing $\mathrm{n}$ molecules. In both cases, $i$ runs over the different trial configurations.

The average binding energy provides information concerning the interaction between water molecules and their surroundings, i. e., the interaction between water molecules and the inner wall of the nanotube via hydrogen bonds (HBs) as well as the intensity of the intermolecular HB interactions formed between water molecules [44, 47]. In turn, the average coupling energy quantifies solely the intermolecular interactions between water molecules and the inner wall of the FFNT [44, 47].

Finally, the unit cell of the relaxed structures containing 20, 21, and 22 water molecules was tripled along the $\mathrm{c}$ direction. Steps 1-3 were repeated with a production run of $12.0 \mathrm{ps}$. The last $10.0 \mathrm{ps}$ were used to calculate the pair distribution functions as well as the number of formed hydrogen bonds.

To compute the number of HBs, we used as a cutoff distance criteria smaller than the position of the first minimum in the pair distribution functions formed between donor and acceptor atoms as well as donated hydrogens and acceptor atoms possibly involved in HBs. Here, we considered oxygen in water, carboxylate and carbonyl oxygens and ammonium nitrogen in FFNT as HB acceptors. Oxygen from water, and ammonium nitrogen (in the FFNTs) are regarded as HB donors. Furthermore, only angles smaller than $30^{\circ}$ between acceptor, donor, and hydrogen atoms were considered.

\section{Results and discussion}

The computed lattice parameters of the hexagonal anhydrous NT ( $a=24.21 \AA, c=5.51 \AA)$ agree with the experimental results [8]. The diameter of the inner channel-the distance between nitrogens in opposing $\mathrm{NH}_{3}^{+}$groups-of $\sim 12.19 \AA$ is also comparable to the one observed by Kim et al. [28].

Once the relaxed anhydrous structure was obtained, the question of whether the FFNT structure is stable in the gas phase arises. Figure 2 shows snapshots of an MD simulation for the anhydrous system starting from zwitterionic building blocks. We notice that after $\sim 1.00 \mathrm{ps,} \mathrm{an}$ intramolecular proton transfer occurs from one of the $\mathrm{NH}_{3}^{+}$ to $\mathrm{COO}^{-}$groups. After $\sim 5.00 \mathrm{ps}$, all the zwitterionic molecules that compose one hexamer change to the canonical neutral form.

The difference in energy per FF molecule between the initial (zwitterionic) and final (neutral) structures was 0.25 $\mathrm{eV}$. While we do not observe a phase transition due to the fixed symmetry (the unit cell does not change shape), the calculations without boundary conditions resulted in structures, which were considerably distorted. This leads to the conclusion that the anhydrous zwitterionic form is a metastable solution. In fact, we also compared the anhydrous structure with the orthorhombic phase experimentally observed at higher temperatures. There, the molecular precursor for the structure is a cyclic form of the FF molecule $[1,31,40]$. Our calculations indicate that it is $0.80 \mathrm{eV}$, per FF molecule, more stable than the zwitterionic form. Thus, as it has been speculated, the removal of water would lead to a hexagonal-to-orthorhombic transition, as the structure becomes more stable [24].

In order to understand how water molecules interact with and possibly stabilize the crystal structure, we trapped a different number of $\mathrm{H}_{2} \mathrm{O}$ molecules inside each hydrophilic 

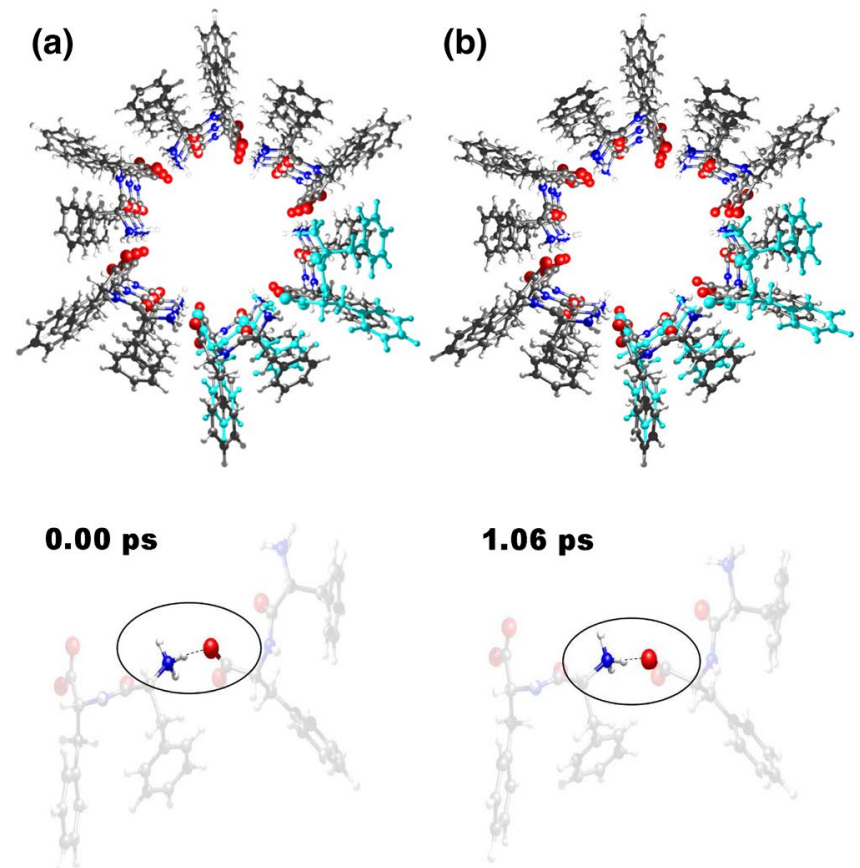

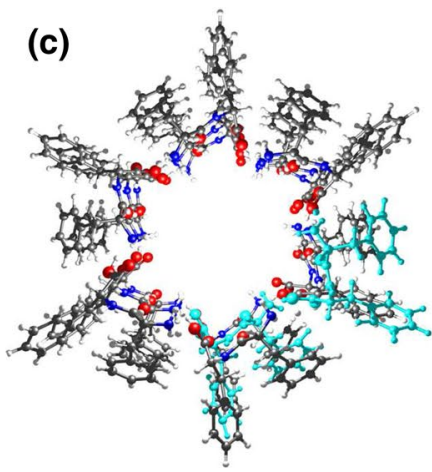

1.06 ps

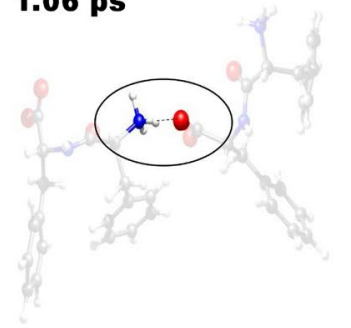

5.07 ps

(d)

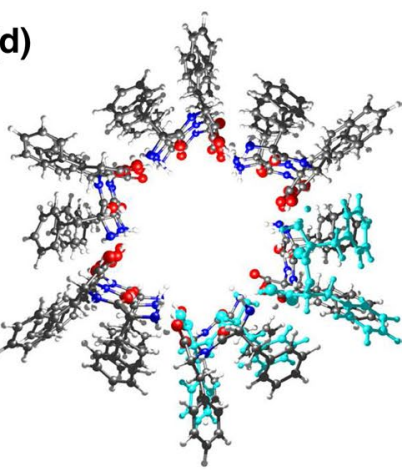

11.00 ps

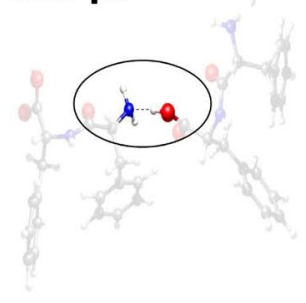

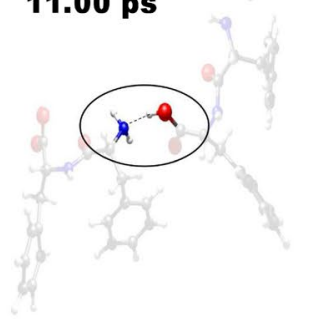

Fig. 2 a Initial configuration of the FFNT and at b 1.06 ps, c 5.07 ps, and d 11.00 ps during the molecular dynamics simulation. One observed representation of two FF molecules (in cyan) involved in

channel: from 1 to 23 molecules per hexamer. The average binding and coupling energies are presented in Fig. 3. In the same figure, we also present the relative energy of the neutral form of the nanotube and the energy of six formula units of the orthorhombic structure. From Fig. 3, the intermolecular water interactions turn all structures into stable ones when compared to the zwitterionic form of the anhydrous NT. We also observe that all the residues keep their zwitterionic structure during the MD simulation-for any number of water molecules which means that a small number of them is already enough to stabilize the tubes. Most interestingly, the minima of $E_{b}$ and $E_{c}$ occur for different concentrations of water (20 molecules for $E_{c}$, and 21 for $E_{b}$ ). That is, by adding an extra water molecule to the system (starting from $n=20$ ), the interaction with the nanotube wall becomes unfavorable. However, the water molecules are arranged in such a way that the interaction between them makes the system more stable until we add another water molecule (for a total of 22), in which case both $E_{b}$ and $E_{c}$ increase.

Increasing threefold the unit cell along the $z$ direction and performing a new MD simulation allowed for the identification of the mechanisms leading to the stability of the nanotubes around the minima. In Fig. 4, we present the time-averaged contour plots of the density of oxygen atoms in water projected on the plane perpendicular to the NT axis (ab-plane) [9]. For the systems with 20 and 21 water the hydrogen transfer process between ammonium and carboxylate groups is shown for each snapshot. It is possible to notice the proton transfer

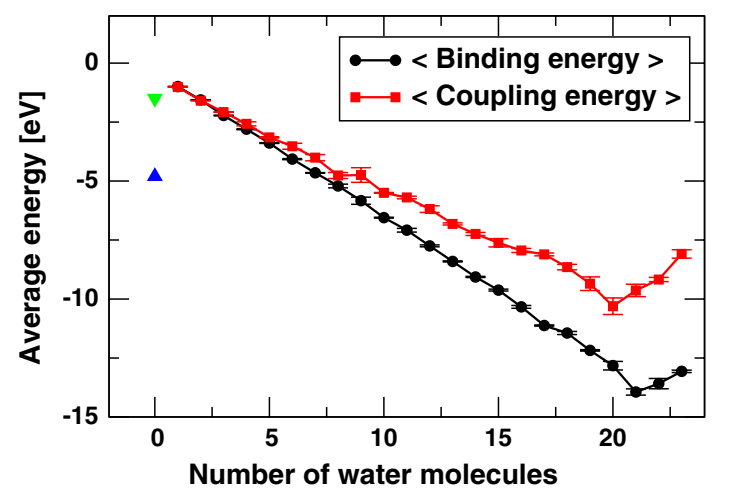

Fig. 3 Average binding and coupling energy as a function of number of water molecules confined in the hydrophilic channel of FFNT for each hexamer. The green down triangle is the energy difference between neutral and zwitterionic structures. The blue up triangle corresponds to the energy difference between the orthorhombic and zwitterionic hexagonal arrangement

molecules per hexamer, a clear hexagonal structure is noted close to the NT wall. As we will show, this is related to the oxygen- and nitrogen-containing functional groups making hydrogen bonds with oxygen atoms in water. With increasing water concentration, the inner shell becomes denser, and the hexagonal structure becomes less clear.

It is worth noting that the voids formed between the first and second shells are greater in the case with 

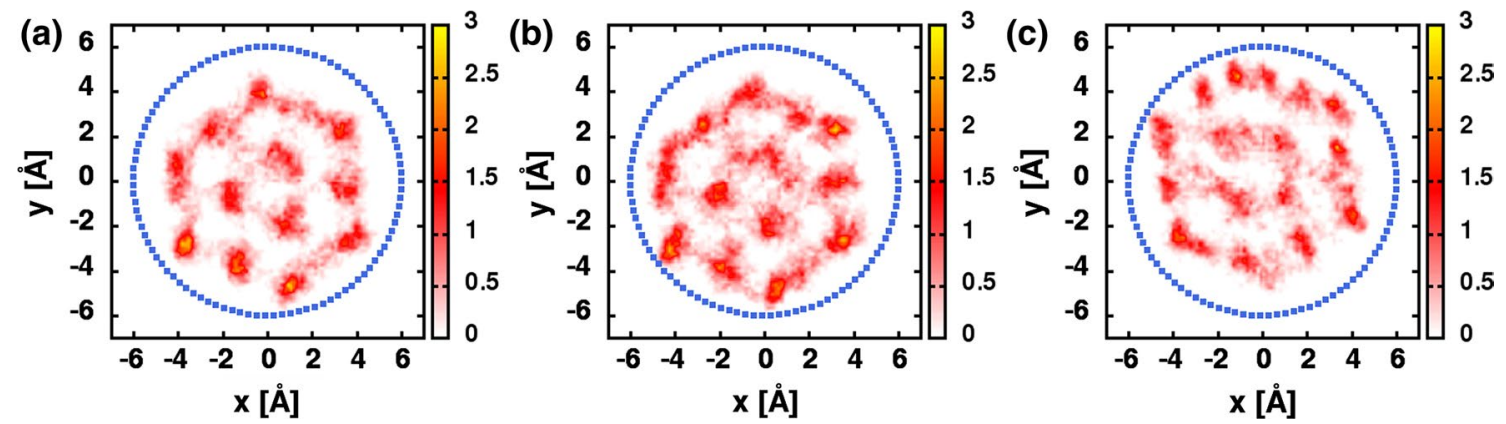

Fig. 4 Contour map of the distribution of oxygen atoms of water molecules projected onto the ab-plane throughout the trajectory for the system with a 20, b 21, and c 22 water molecules per hexamer

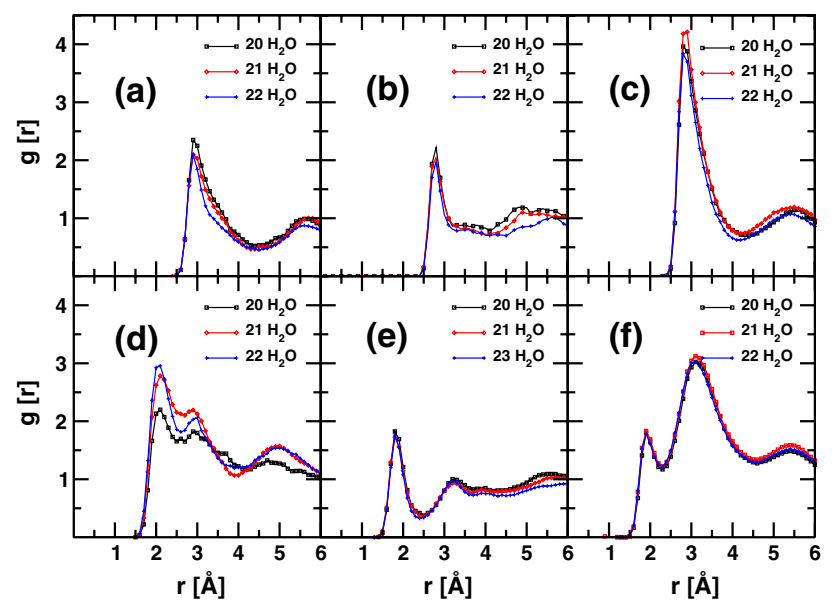

Fig. 5 Pair distribution functions formed between a) $\mathrm{N}_{N H_{3}^{+}}-\mathrm{O}_{W}$, b) $\mathrm{O}_{\mathrm{COO}}-\mathrm{O}_{W}$, c) $\mathrm{O}_{W}-\mathrm{O}_{W}$, d) $\mathrm{O}_{W}-\mathrm{H}_{\mathrm{NH}_{3}^{+}}$, e) $\mathrm{O}_{\mathrm{COO}}-\mathrm{H}_{W}$ and f) $\mathrm{O}_{W}-\mathrm{H}_{W}$

20 than the one with 21 water molecules per hexamer. That is, water molecules have more space to accommodate avoiding strong repulsive interactions between them. It shows that the water molecules tend to bind to the surface of the tube before forming HBs among themselves.

The addition of extra water molecules produces a broader hexagonal solvation shell as well as a denser second shell. One can see in Fig. 4c that the voids formed between the first and second shells become smaller. Consequently, the steric hindrance between water molecules is higher than the other two lower concentrations. Then, the water-water interactions become repulsive, thus not being able to stabilize the NT water system.

The pair distribution functions (PDF) are shown in Fig. 5. There, we present the PDFs formed between donors and acceptors (Fig. 5a-c): ammonium nitrogen and water oxygen atoms $\left(\mathrm{N}_{\mathrm{NH}_{3}^{+}}-\mathrm{O}_{\mathrm{W}}\right)$, oxygen atoms of water and carboxylate groups $\left(\mathrm{O}_{\mathrm{COO}^{-}}-\mathrm{O}_{\mathrm{W}}\right)$, and between water
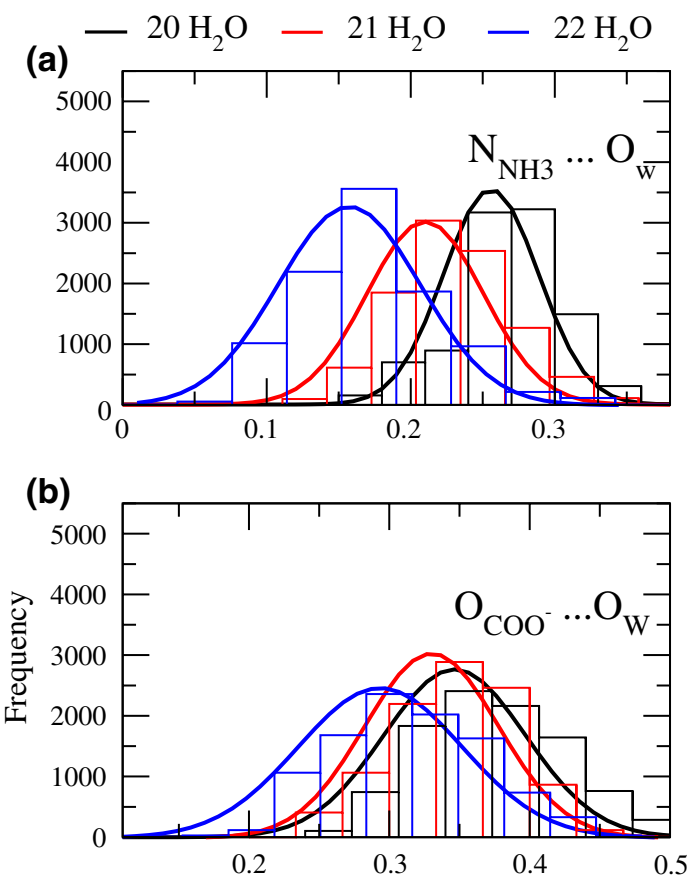

(c)

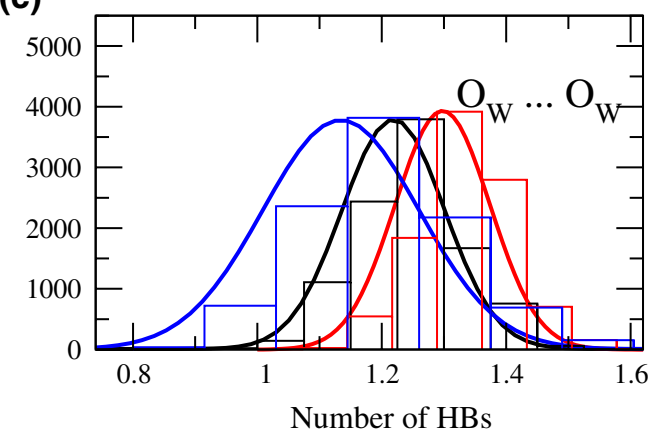

Fig. 6 Distribution of the number of HBs formed during the MD simulations for different water content per hexamer: a ammonium nitrogen and water oxygen, b carboxylate oxygen and water oxygen, and $\mathbf{c}$ water oxygens

oxygen atoms $\left(\mathrm{O}_{\mathrm{W}}-\mathrm{O}_{\mathrm{W}}\right)$. Furthermore, in Fig. 5d-f, we present the PDFs formed between acceptor groups and 
donated hydrogens: water oxygens and extra hydrogen atoms in ammonium $\left(\mathrm{O}_{\mathrm{W}}-\mathrm{H}_{\mathrm{NH}_{3}^{+}}\right)$, water hydrogens and carboxylate oxygens $\left(\mathrm{O}_{\mathrm{COO}^{-}}-\mathrm{H}_{\mathrm{W}}\right)$, and hydrogen atoms and oxygen in water $\left(\mathrm{O}_{\mathrm{W}}-\mathrm{H}_{\mathrm{W}}\right)$.

A broad first peak in the $\mathrm{N}_{\mathrm{NH}_{3}^{+-}} \mathrm{O}_{\mathrm{W}} \mathrm{PDF}$ is found at approximately $3.07 \AA$. Similarly, one can note that the first peaks in the $\mathrm{O}_{\mathrm{W}}-\mathrm{H}_{\mathrm{NH}_{3}^{+}}$PDFs are broad. However, the $\mathrm{O}_{\mathrm{W}}-\mathrm{H}_{\mathrm{NH}_{3}^{+}} \mathrm{PDFs}$ are split into two. The formation of two peaks is related to the formation of two unlike water oxygen first nearest neighbors, which accept the additional ammonium hydrogen. Thus, one can conclude that the water molecules are randomly oriented around $\mathrm{NH}_{3}^{+}$groups. On the other hand, $\mathrm{O}_{\mathrm{COO}^{-}}-$water PDFs demonstrate that water molecules are more oriented around the $\mathrm{COO}^{-}$groups than the ammonium motifs, as a first sharp peak located at 2.82 $\AA(1.81 \AA)$ of the $\mathrm{O}_{\mathrm{COO}^{-}}-\mathrm{O}_{\mathrm{W}}\left(\mathrm{O}_{\mathrm{COO}^{-}}-\mathrm{H}_{\mathrm{W}}\right)$ PDF can be seen in Fig. 5b, e. Important features concerning the $\mathrm{O}_{\mathrm{W}}$ $-\mathrm{O}_{\mathrm{W}}$ PDFs are noticed in Fig. 5c. The tetrahedral arrangement formed by the water molecules involved in HB is lost. This is because the first peak in the $\mathrm{O}_{\mathrm{W}}-\mathrm{O}_{\mathrm{W}}$ PDFs, located at $2.83 \AA$, is not sharp as observed in bulk, and the second peak is indeed shifted to $5.35 \AA$. It is worth noting, with regard to the second peak, that its intensity is reduced compared to the bulk case $[14,37]$. The HB distance in the $\mathrm{O}_{\mathrm{W}}$ $-\mathrm{H}_{\mathrm{W}}$ PDFs shown in Fig. $5 \mathrm{f}$ is shifted to $1.90 \AA$ A. It means that some HB distances in water molecules have moved to longer distances [14, 37]; an indication that the structure of water confined inside the channel is significantly different $[7,49]$. This is directly related to the excluded volume effect imposed by the hydrophilic groups of FFNTs, which are particular sets of donor and acceptor sites involved in hydrogen bonds with the confined system [7, 49].

Finally, from our definition of hydrogen bond, we present in Fig. 6 the average number of HBs formed in the complex. We can note that from 20 to 22 water molecules per hexamer, the number of HBs between water molecules and oxygen atoms of $\mathrm{COO}^{-}$as well as $\mathrm{NH}_{3}^{+}$groups decreases monotonically. Contrary to what was previously though, our results indicate that the interaction is prominent between the carboxylate group and water [19]. For 21 water molecules, by breaking HBs formed between water and the atoms of the NT wall, the solvent increases the number of these intermolecular interactions in an attempt to restabilize the complex. Furthermore, the total number of $\mathrm{HB}$ is similar to those observed in other confined water systems [39].

In fact, one can note that the intermolecular interaction between water molecules is stronger than the one between the solvent and the corresponding atoms of the NT wall. For the latter case, besides the reduction in the number of
HBs between NT and water, increasing the density of the solvent within the hydrophilic channel provides a drastic reduction in the number of HBs formed between them.

\section{Conclusions}

The stability of peptide nanotubes formed by FF molecules in zwitterionic form with and without water molecules under confinement was considered in this work using DFTB molecular dynamics simulations. We showed that the anhydrous zwitterionic system is a metastable solution. In fact, the presence of temperature effects during the calculations leads to a zwitterionic to neutral form transition deforming the nanotube. We also showed that the orthorhombic phase is the most stable one in the absence of water, which ultimately could explain the phase transitions observed in experiments [1, 24, 31]. We showed that a small amount of water molecules is enough to stabilize the structure, and that the molecules tend to form a hexagonal structure that follows the arrangement of the FFNT walls. Thus, the stability of the nanotube is closely intertwined with the presence of water. Finally, our analysis indicates that the stability of the tube arises from a combination of hydrogen bonds formed between ammonia and carboxylate groups and water, and between water molecules. While the hydrogen bonds with the wall of the NT are significant, when steric effects start to play a role, the water molecules rearrange themselves to allow for the formation of extra hydrogen bonds between water molecules, and keep the tube stable.

Acknowledgments We thank L. S. Pedroza, R. Gester, and J. Guimarães for fruitful discussions. We acknowledge financial support from FAPESP (Grant \# 2013/12997-0), CNPq (Grants \# 472197/2012-6 and 305186/2012-4), PAPIM (Grant \# 63012906), PROINT (Grant \# 128001208048), PARD (Grant \# 1563012909), and INCT in Bioanalytics (FAPESP, Grant 2008/57805-2; and CNPq, Grant 573672/2008-3). A. R. R. acknowledges support from CNPq (Grants \# 482021/2013-6 and 558205/2010-0), FAPESP (Grant \# 2013/02112-0), ICTP-SAIFR (FAPESP project 2011/11973-4) and the ICTP-Simons Associate scheme. Computational support was provided by NACAD/UFRJ, CENAPAD/UNICAMP, CESUP/UFRGS, GridUNESP/UNESP, and the HPC center at UFABC.

\section{References}

1. Adler-Abramovich L, Aronov D, Beker P, Yevnin M, Stempler S, Buzhansky L, Rosenman G, Gazit E (2009) Self-assembled arrays of peptide nanotubes by vapour deposition. Nat Nanotech 4:849-854

2. Amdursky N, Beker P, Koren I, Bank-Srour B, Mishina E, Semin S, Rasing T, Rosenberg Y, Barkay Z, Gazit E, Rosenman G (2011) Structural transition in peptide nanotubes. Biomacromolecules 12:1349-1354 
3. Andersen HC (1980) Molecular dynamics simulations at constant pressure and/or temperature. J Chem Phys 72:2384-2393

4. Andrade-Filho T, Ferreira FF, Alves WA, Rocha AR (2013) The effects of water molecules on the electronic and structural properties of peptide nanotubes. Phys Chem Chem Phys 15:7555-7559

5. Aradi B, Hourahine B, Frauenheim T (2007) DFTB+, a sparse matrix-based implementation of the DFTB method. J Phys Chem A 111:5678-5684

6. Bianchi RC, da Silva ER, Dall'Antonia LH, Ferreira FF, Alves WA (2014) A nonenzymatic biosensor based on gold electrodes modified with peptide self-assemblies for detecting ammonia and urea oxidation. Langmuir 30(38):11,464-11,473

7. Calero S, Gómez-Álvarez P (2014) Effect of the confinement and presence of cations on hydrogen bonding of water in LTAtype zeolite. J Phys Chem C 118:9056-9065

8. Cipriano TC, Takahashi PM, de Lima D, Oliveira VX, Souza JA, Martinho H, Alves WA (2009) Spatial organization of peptide nanotubes for electrochemical devices. J Mater Sci 45:5101-5108

9. Clark JK, Paddison SJ, Clark JK II, Paddison SJ (2014) Ab initio molecular dynamics simulations of water and an excess proton in water confined in carbon nanotubes. Phys Chem Chem Phys $16: 17,756-17,769$

10. Djalali R, Samson J, Matsui H (2004) Doughnut-shaped peptide nano-assemblies and their applications as nanoreactors. J Am Chem Soc 126:7935-7939

11. Do TD, Bowers MT (2015) Diphenylalanine self assembly: novel ion mobility methods showing the essential role of water. Anal Chem 87:4245-4252

12. Elstner M, Porezag D, Jungnickel G, Elsner J, Haugk M, Frauenheim T, Suhai S, Seifert G (1998) Self-consistent-charge density-functional tight-binding method for simulations of complex materials properties. Phys Rev B 58:7260-7268

13. Fairman R, Akerfeldt K (2005) Peptides as novel smart materials. Curr Opin Struct Biol 15:453-463

14. Gallo P, Rovere M (2003) Anomalous dynamics of confined water at low hydration. J Phys Condens Matter 15:7625-7633

15. Gaus M, Cui Q, Elstner M (2011) DFTB3: extension of the selfconsistent-charge density-functional tight-binding method (SCCDFTB). J Chem Theory Comput 7:931-948

16. Gaus M, Goez A, Elstner M (2013) Parametrization and benchmark of DFTB3 for organic molecules. J Chem Theory Comput 9:338-354

17. Ghadiri MR, Granja JR, Milligan RA, McRee DE, Khazanovich N (1993) Self-assembling organic nanotubes based on a cyclic peptide architecture. Nature 366:324-327

18. Görbitz CH (2003) Nanotubes from hydrophobic dipeptides: pore size regulation through side chain substitution. New J Chem 27:1789-1793

19. Görbitz CH (2007) Microporous organic materials from hydrophobic dipeptides. Chem Eur J 13:1022-1031

20. Guo C, Luo Y, Zhou R, Wei G (2012) Probing the self-assembly mechanism of diphenylalanine-based peptide nanovesicles and nanotubes. ACS Nano 6:3907-3918

21. Han TH, Kim J, Park JS, Park CB, Ihee H, Kim SO (2007) Liquid crystalline peptide nanowires. Adv Mater 19:3924-3927

22. Han TH, Oh JK, Park JS, Kwon SH, Kima SW, Kim SO (2009) Highly entangled hollow $\mathrm{TiO}_{2}$ nanoribbons templating diphenylalanine assembly. J Mater Chem 19:3512-3516

23. Hartgerink JD, Granja JR, Milligan RA, Ghadiri MR (1996) Self-assembling peptide nanotubes. J Am Chem Soc 118:43-50

24. Heredia A, Bdikin I, Kopyl S, Mishina E, Semin S, Sigov A, German K, Bystrov V, Gracio J, Kholkin AL (2010) Temperaturedriven phase transformation in self-assembled diphenylalanine peptide nanotubes. J Phys D: Appl Phys 43:462,001-462,006
25. Im S, Jang SW, Lee S, Lee Y, Kim B (2008) Arginine zwitterion is more stable than the canonical form when solvated by a water molecule. J Phys Chem A 112:9767-9770

26. Jeon J, Mills CE, Shell MS (2013) Molecular insights into diphenylalanine nanotube assembly: all-atom simulations of oligomerization. J Phys Chem B 117:3935-3943

27. Kelly CM, Northey T, Ryan K, Brooks BR, Kholkin AL, Rodriguez BJ, Buchete NV (2015) Conformational dynamics and aggregation behavior of piezoelectric diphenylalanine peptides in an external electric field. Biophys Chem 196:16-24

28. Kim J, Han TH, Kim YI, Park JS, Choi J, Churchill DG, Kim SO, Ihee H (2010) Role of water in directing diphenylalanine assembly into nanotubes and nanowires. Adv Mater 22:583-587

29. Kim JY, Ahn DS, Park SW, Lee S (2014) Gas phase hydration of amino acids and dipeptides: effects on the relative stability of zwitterion vs. canonical conformers. RSC Adv $4: 16,352-16,361$

30. Kogikoski S, Sousa CP, Liberato MS, Andrade-Filho T, Prieto T, Ferreira FF, Rocha AR, Guha S, Alves WA (2016) Multifunctional biosensors based on peptide-polyelectrolyte conjugates. Phys Chem Chem Phys 18:3223-3233

31. Lee JS, Yoon I, Kim J, Ihee H, Kim B, Park CB (2011) Selfassembly of semiconducting photoluminescent peptide nanowires in the vapor phase. Angew Chem Int Ed 50:1164-1167

32. Martínez JM, Martínez L (2003) Packing optimization for automated generation of complex system's initial configurations for molecular dynamics and docking. J Comput Chem 24:819-825

33. Martínez L, Andrade R, Birgin EG, Martínez JM (2009) Packmol: a package for building initial configurations for molecular dynamics simulations. J Comput Chem 30:2157-2164

34. Monkhorst HJ, Pack JD (1976) Special points for Brillouin-zone integrations. Phys Rev 13:5188-5192

35. Reches M, Gazit E (2003) Casting metal nanowires within discrete self-assembled peptide nanotubes. Science 300:625-627

36. Reches M, Gazit E (2006) Molecular self-assembly of peptide nanostructures: mechanism of association and potential uses. Curr Nanosci 2:105-111

37. Rovere M, Ricci MA, Vellati D, Bruni F (1998) A molecular dynamics simulation of water confined in a cylindrical $\mathrm{SiO}_{2}$ pore. J Chem Phys 108:9859-9867

38. Ryu J, Kim SW, Kang K, Park CB (2009) Synthesis of diphenylalanine/cobalt oxide hybrid nanowires and their application to energy storage. ACS Nano 4:159-164

39. Soper AK, Bruni F, Ricci MA (1998) Water confined in Vycor glass. II. Excluded volume effects on the radial distribution functions. J Chem Phys 109:1486-1494

40. Souza MI, Silva ER, Jaques YM, Ferreira FF, Fileti EE, Alves WA (2014) The role of water and structure on the generation of reactive oxygen species in peptide/hypericin complexes. J Pept Sci 20:554-562

41. Tortonda FR, Pascual-Ahuir JL, Silla E, Tuñón I, Ramírez FJ (1998) Aminoacid zwitterions in solution: geometric, energetic, and vibrational analysis using density functional theory-continuum model calculations. J Chem Phys 109(2):592-603

42. Vauthey S, Santoso S, Gong H, Watson N, Zhang S (2002) Molecular self-assembly of surfactant-like peptides to form nanotubes and nanovesicles. Proc Nat Acad Sci 99:5355-5360

43. Wang J, Liu K, Yan L, Wang A, Bai S, Yan X (2016) Trace solvent as a predominant factor to tune dipeptide self-assembly. ACS Nano 10:2138-2143

44. Wang L, Zhao J, Li F, Fang H, Lu JP (2009) First-principles study of water chains encapsulated in single-walled carbon nanotube. J Phys Chem C 113:5368-5375

45. Wang M, Xiong S, Wu X, Chu PK (2011) Effects of water molecules on photoluminescence from hierarchical peptide nanotubes and water probing capability. Small 7:2801-2807 
46. Yan X, He Q, Wang K, Duan L, Cui Y, Li J (2007) Transition of cationic dipeptide nanotubes into vesicles and oligonucleotide delivery. Angew Chem Int Ed 46:2431-2434

47. Yang R, Hilder TA, Chung SH, Rendell A (2011) First-principles study of water confined in single-walled silicon carbide nanotubes. J Phys Chem C 115:17,255-17,264

48. Yemini M, Reches M, Gazit E, Rishpon J (2005) Peptide nanotube-modified electrodes for enzyme-biosensor applications. Anal Chem 77:5155-5159
49. Youssef M, Pellenq RJM, Yildiz B (2011) Glassy nature of water in an ultraconfining disordered material: the case of calcium-silicate-hydrate. J Am Chem Soc 133:2499-2510

50. Zhang J, Wu X, Gan Z, Zhu X, Jin Y (2014) Unidirectionally aligned diphenylalanine nanotube/microtube arrays with excellent supercapacitive performance. Nano Res 7:929-937

51. Zhang S (2003) Fabrication of novel biomaterials through molecular self-assembly. Nat Biotech 21:1171-1178 